\title{
Early implementation of protective measures defines surgical outcomes in the COVID-19 pandemic
}

\author{
Ana Senent-Boza ${ }^{1}$ (D) Inmaculada Benítez-Linero ${ }^{2} \cdot$ Luis Tallón-Aguilar $^{1} \cdot$ Alejandro Sánchez-Arteaga $^{1}$. \\ Lidia Melero-Cortés ${ }^{3} \cdot$ Felipe Pareja-Ciuró ${ }^{1} \cdot$ Javier Padillo-Ruiz ${ }^{1,4}$
}

Received: 4 May 2020 / Accepted: 14 June 2020 / Published online: 22 July 2020

(c) Springer Nature Singapore Pte Ltd. 2020

\begin{abstract}
Quick implementation of specific protocols and protective measures in a tertiary hospital in Spain allowed for the early diagnosis and optimal management of patients with SARS-CoV-2 infection and proper protection of staff and inpatients. From the COVID-19 outbreak in this country until the time of writing, 14 patients in our hospital underwent surgery with COVID-19, or COVID-19 developed postoperatively. Their postoperative outcomes did not differ from those in our routine clinical practice, with a $0 \%$ respiratory failure rate and a $7.14 \%$ mortality rate, in contrast with other published series. COVID19 did not develop in any of the healthcare workers present in the operating room during these procedures or in those who cared for these patients on the ward.
\end{abstract}

Keywords Surgical outcomes · COVID-19 - SARS-CoV-2

\section{Introduction}

The global crisis triggered by the new coronavirus that emerged at the end of 2019 [1], known as severe acute respiratory syndrome coronavirus 2 (SARS-CoV-2), has undoubtedly changed healthcare worldwide. There is lack of evidence about the proper management of surgical patients in this context and the data published about the influence of coronavirus disease 2019 (COVID-19) on postoperative outcomes are inconsistent and derived from short case series $[2,3]$. To avoid hospital collapse, isolate hospital beds for patients with COVID-19 and prevent a higher complication rate in surgical patients, the trend in areas with the highest incidence of COVID-19 has been to delay most elective surgery, with the exception of non-deferrable surgery

Ana Senent-Boza

asenentboza@gmail.com

1 Department of General and Digestive Surgery, Virgen del Rocio University Hospital, Avda. Manuel Siurot s/n, PC 41013 Seville, Spain

2 Department of Anesthesiology and Reanimation, Virgen del Rocio University Hospital, Seville, Spain

3 Department of Gynecology and Obstetrics, Virgen del Rocio University Hospital, Seville, Spain

4 Professor of Surgery, University of Seville, Seville, Spain such as oncologic procedures. However, acute conditions needing urgent surgery cannot be avoided and these patients may have concomitant SARS-CoV-2 infection. When a surgical procedure cannot be postponed, early diagnosis of the SARS-CoV-2 infection is crucial, not only for optimal treatment and follow-up of the patient, but also for protecting healthcare professionals, and accordingly, preventing the spread of the virus.

\section{Protective measures, perioperative COVID-19 diagnosis and postoperative outcomes}

\section{Context}

The first case of confirmed COVID-19 in the city of Seville and the Andalusian region, Spain, was reported on February 26th, 2020, by our hospital. Spanish lockdown was declared on March 16th, 2020. At that time, there were 19 patients with confirmed SARS-CoV-2 infection in our hospital area, 7 of whom were admitted. Since then, 14 patients (50\% women) with a median age of 66.5 years (range 33-85 years), have undergone a surgical procedure with highly suspected or confirmed COVID-19, or the disease developed during the postoperative period and before 
discharge. These procedures were conducted by general and gastrointestinal surgeons (7), gynecologist-obstetricians (2), orthopedic surgeons (2), interventional radiologists (1), otorhinolaryngologists (1) and neurosurgeons (1).

\section{Implementation of protective measures and COVID-19 screening protocol}

Aiming to avoid in-hospital transmission and staff infection, several measures were implemented early to enforce social and working distancing and allow for the early detection of COVID-19 cases, including telematic clinical sessions and committees, telephonic review for outpatients, postponement of deferrable elective surgery, the designation of specific COVID-19 areas and pathways and the development of COVID-19 screening protocols and clinical and surgical management guidelines. We developed locally adapted protocols that are being updated daily in line with evolving evidence [4]. Table 1 summarizes all the protective measures that were taken.

To increase the detection rate of SARS-CoV-2-infected patients, exhaustive clinical screening has been carried out. All patients are specifically interrogated for typical COVID19 symptoms (fever, dry cough, dyspnea, fatigue, anosmia and ageusia) and risk contacts before any surgical procedure, whether in the emergency setting or before any non-deferrable elective surgery, and daily during the postoperative stay. This clinical screening included relatives and other persons accompanying the patients. Reverse transcriptase polymerase chain reaction (RT-PCR) tests for SARS-CoV-2 were performed if there was any clinical suspicion.

From March 26th, this screening was reinforced with a preoperative chest computed tomography (CCT) [4]. If radiological findings of COVID-19 were identified, a nasopharyngeal sample was collected and sent for SARS-CoV-2 detection by RT-PCR. However, emergency procedures were not delayed until test result confirmation (5-6 $\mathrm{h}$ from the sampling time) and non-deferrable procedures were performed with the same protective measures implemented as for positive cases. If COVID-19 was suspected or confirmed, elective surgery was not performed.

The personal protective equipment (PPE) worn by the medical staff involved in surgical procedures on patients with COVID-19 included disposable FFP2 masks and shoe covers, googles, visors, caps, double gloves and waterproof gowns. PPE donning and doffing was completed with the buddy system, following a checklist approved by the Department of Preventive Medicine [4]. To prevent aerosol generation, regional anesthesia was selected over general anesthesia whenever possible, and an open approach was selected over a laparoscopic approach. Intubation was performed by videolaryngoscopy when possible. For minimally invasive procedures, filtering devices for $\mathrm{CO} 2$ evacuation were available. COVID-19 has not developed in any of the healthcare workers or other staff present in the operating room during these surgical procedures and positive COVID-19 cases among ward staff are anecdotal, at under $2 \%$.

\section{Perioperative COVID-19 diagnosis}

Eight patients underwent surgery after confirmation or with suspected COVID-19, based on the development of typical symptoms from 14 to 2 days beforehand. None of the patients were in respiratory failure or required oxygen supplementation at the time of the procedures. All surgeries performed were non-deferrable based on the urgency of the diagnosis, such as intestinal perforation, ruptured ectopic pregnancy, or strangulated femoral hernia. Elective procedures were not performed in patients with suspected or confirmed COVID-19. COVID-19 infection was

Table 1 Protective measures in surgical areas during the COVID-19 pandemic

\begin{tabular}{|c|c|c|c|}
\hline Clinical & Logistical & Department organization & Educational \\
\hline $\begin{array}{l}\text { Cancellation of all deferrable } \\
\text { elective procedures }\end{array}$ & $\begin{array}{l}\text { Designation of COVID-19 treat- } \\
\text { ment areas in operating room, } \\
\text { ICU and wards }\end{array}$ & Rotating staff shifts & $\begin{array}{l}\text { Cessation of new observerships and } \\
\text { fellowships }\end{array}$ \\
\hline $\begin{array}{l}\text { Daily clinical screening for } \\
\text { COVID-19 to all inpatients }\end{array}$ & $\begin{array}{l}\text { Designation of specific trans- } \\
\text { fer pathways for COVID-19 } \\
\text { patients }\end{array}$ & Telematic clinical committees & $\begin{array}{l}\text { Discontinuation of resident rota- } \\
\text { tions }\end{array}$ \\
\hline $\begin{array}{l}\text { Preoperative chest } \mathrm{CT} \pm \mathrm{RT}-\mathrm{PCR} \\
\text { screening for COVID-19 }\end{array}$ & $\begin{array}{l}\text { Check-list for PPE donning and } \\
\text { doffing for surgical procedures } \\
\text { on COVID-19 patients }\end{array}$ & $\begin{array}{l}\text { Early preventive isolation of staff } \\
\text { with risk contacts or COVID-19 } \\
\text { symptoms }\end{array}$ & $\begin{array}{l}\text { Discontinuation of clinical rotations } \\
\text { of medical students }\end{array}$ \\
\hline Telephonic review for outpatients & $\begin{array}{l}\text { Specific decontamination proto- } \\
\text { cols for COVID-19 areas and } \\
\text { surgical instruments used }\end{array}$ & Working distancing (> 2metres) & $\begin{array}{l}\text { Cessation of all face-to-face } \\
\text { educational activities (courses, } \\
\text { congresses...) }\end{array}$ \\
\hline $\begin{array}{l}\text { Development of specific COVID- } \\
19 \text { management guidelines }\end{array}$ & $\begin{array}{l}\text { Limitation of visits and patients' } \\
\text { companions }\end{array}$ & $\begin{array}{l}\text { Cancellation of all vacation } \\
\text { permits }\end{array}$ & $\begin{array}{l}\text { Telematic conferences and educa- } \\
\text { tional webinars }\end{array}$ \\
\hline
\end{tabular}

$C T$ computed tomography, COVID-19 coronavirus disease 2019, ICU intensive care unit, $P P E$ personal protective equipment 
confirmed by RT-PCR in five of these eight patients. One of the three patients with negative RT-PCR test results presented 2 weeks before surgery with symptoms suggestive of COVID-19 and another had highly suggestive COVID19 radiological images. Patients confirmed as positive for SARS-CoV-2 were treated in the COVID-19 isolation ICU or ward according to their clinical situation.

Following our adapted COVID-19 protocol [4], clinical screening was performed daily for all inpatients in the surgical ward and their visitors during their postoperative stay. If any suggestive symptom was reported, patients were RT-PCR tested for SARS-CoV-2. Five patients reported the development of symptoms between 2 and 28 days after surgery and one patient was tested after close contact with a positive case. SARS-CoV2 infection was confirmed by RTPCR in five patients during their postoperative stay. Table 2 summarizes the demographic, clinical and surgical data of these patients, including timing of the COVID-19 diagnosis, tests results and postoperative outcomes.

\section{COVID-19 symptoms}

In our series, only one patient (case 11) was asymptomatic and COVID-19 diagnosis was confirmed after close contact with a visiting relative whose RT-PCR test was confirmed positive during her hospital stay. The most frequent symptoms were fever over $37.5^{\circ} \mathrm{C}(98.86 \%)$, cough $(64.29 \%)$, fatigue $(2.4 \%)$ and mild dyspnea (21.4\%). Other less frequent symptoms are listed in Table 2.

\section{Laboratory findings}

The most notable laboratory findings in our case series were lymphopenia (less than 1000 cells/uL) and high levels of ferritin (above $150 \mathrm{ng} / \mathrm{ml}$ ). Eight of the 14 patients (57.14\%) had lymphopenia at the time of COVID-19 diagnosis and nine of ten patients tested for ferritin (90\%) had high levels (Table 2).

\section{Radiology findings}

Chest radiography was performed in 10 of the 14 patients (71.43\%), with normal findings in $3(30 \%)$, interstitial pattern in $2(20 \%)$, opacities/consolidation in $4(40 \%)$ and pleural effusion in 2 (20\%). CCT was performed in 7 of the 14 patients (50\%), revealing abnormal findings in all 7 , including the characteristic bilateral ground glass opacity in $5(71.4 \%)$ and bilateral tree-in-bud pattern and pleural effusion in the other $2(28.57 \%)$.

\section{COVID-19-specific treatment}

All patients were managed jointly by the surgical team and infectious diseases specialists. Specific medical treatment for COVID-19 followed the official recommendations available for the diagnosis. Hydroxychloroquine was prescribed for patients with pneumonia and/or RT-PCR confirmed SARSCoV-2 infection (9/14 patients, 62.3\%). Lopinavir plus ritonavir was the selected antiviral treatment prescribed, only for patients with pneumonia $(8 / 14,57.1 \%)$. The indication for antibiotic treatment was related to the surgical diagnosis and/ or presence of pneumonia and was prescribed for 13 of the 14 patients $(92.9 \%)$.

\section{Postoperative outcomes}

The severity and complexity of the procedures was classified into three grades: minor (grade 1), intermediate (grade 2) and major or complex (grade $3 / 3+$ ), according to the preoperative test guidelines of the NHS [5]. Ten patients (71.42\%) underwent grade $3 / 3+$ procedures, $3(21.43 \%)$ underwent grade 2 procedures and only $1(7.14 \%)$ underwent a grade 1 procedure. The median operative time was $115 \mathrm{~min}$. The median decrease in hemoglobin levels after surgery was $1.0 \mathrm{~g} / \mathrm{dL}$.

After the procedure, five patients (35.7\%) were transferred directly to ICU in accordance with the surgical process. All these patients were extubated in the operating room or during the initial postoperative hours. The median ICU stay was 1 day (1-6). Pneumonia developed postoperatively in 8 of the 14 patients (57.1\%), but none suffered acute respiratory distress syndrome (ARDS) or respiratory failure or required mechanical ventilation. The severity of COVID-19 disease was mild or moderate in all patients. The incidence of pneumonia was higher in patients who underwent grade $1 / 2$ procedures $(75 \%)$ than in those who underwent grade $3 / 3+$ procedures $(50 \%)$. Non-pulmonary complications were related to the surgical procedure, such as anastomotic dehiscence or bile leak. None of the patients suffered acute kidney or cardiac failure. Seven of the 14 patients $(50 \%)$ had an uneventful postoperative course, excluding pneumonia.

The median postoperative stay was 17 days (4-61). One patient died of stage IV pancreatic cancer and did not suffer pneumonia in the perioperative period. This represents a mortality rate in our series of $7.14 \%$. At the time of writing, after a median follow-up of 69 days (range 20-91 days), no thrombotic events of any kind had developed in any of the patients.

\section{Discussion}

Our experience highlights the essential perioperative considerations when performing any surgical procedure in the context of the COVID-19 outbreak: 


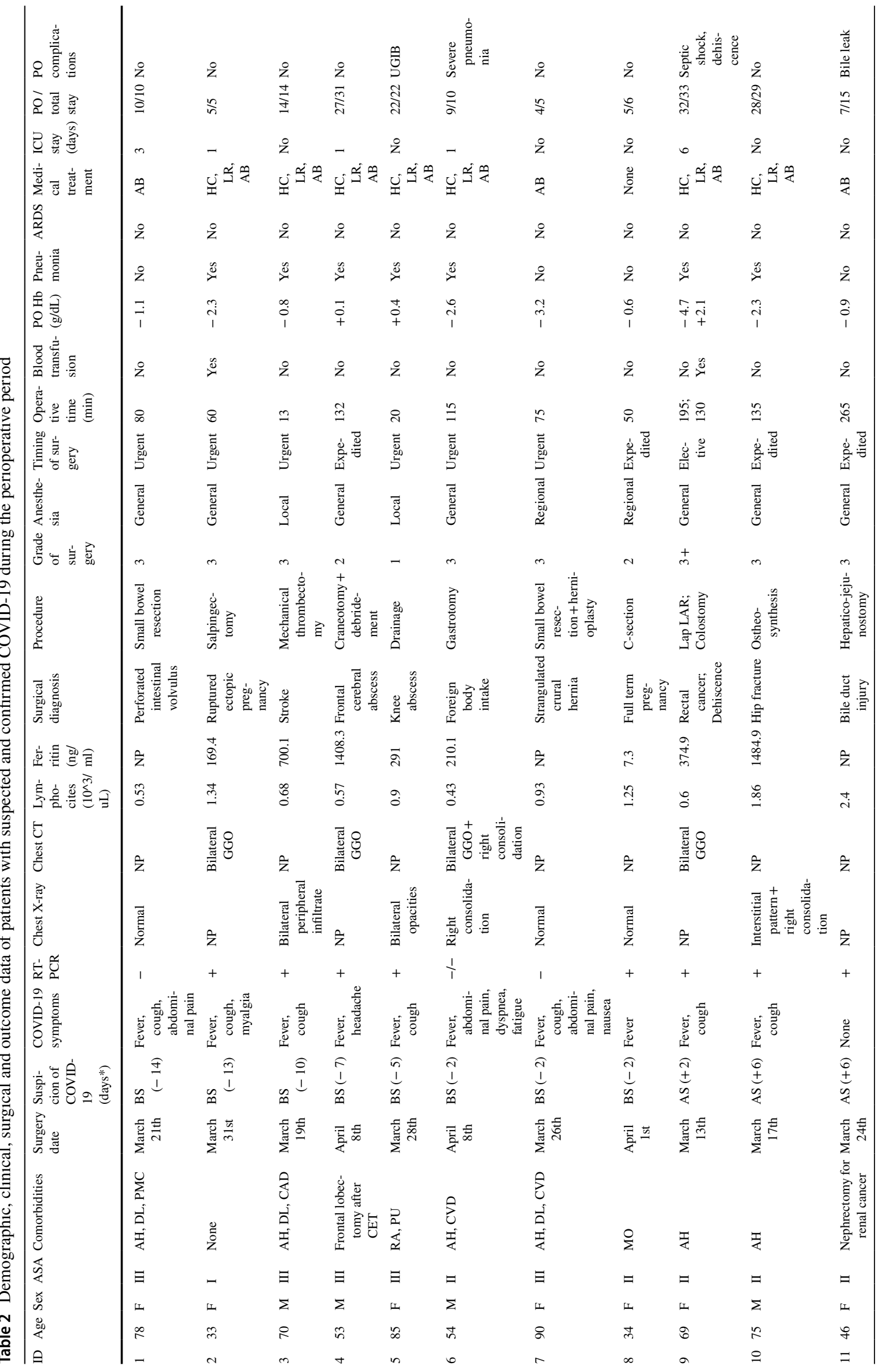




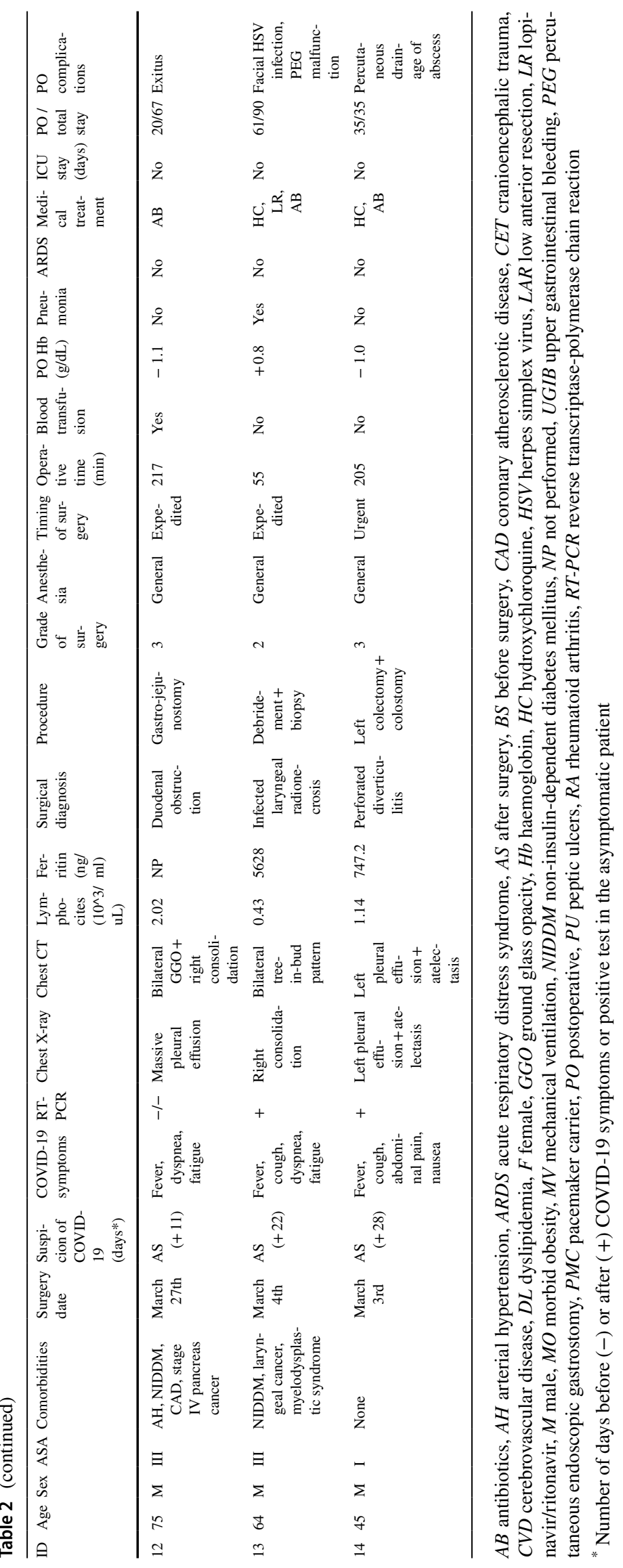


- Early detection of COVID-19 cases is crucial to isolate patients promptly and implement effective protection measures by staff to prevent in-hospital transmission [6]. Specific clinical screening for COVID-19 should be performed in all patients undergoing surgery. The combination of CCT and RT-PCR, depending on the available resources, may increase the rate of detection of positive patients. CCT has been reported by some studies to have higher sensitivity than RT-PCR for the diagnosis of COVID-19 [7]. In our series, two patients with typical radiological images had a repeatedly negative RT-PCR test result. Another patient, without suggestive radiological imaging and a negative RT-PCR test, reported COVID-19 symptoms 14 days before surgery, demonstrating that the RT-PCR can negativize after 3-19 days [8]. When available, the IgM/IgG test may help to confirm or exclude SARS-CoV-2 infection, always considering its low sensitivity (64\% regardless of evolution time, $80 \%$ after 7 days [9]). IgM/IgG tests were not initially available in our hospital.

- To prevent in-hospital transmission and staff infection, it is decisive to promptly implement protective and distancing measures, such as the postponement of elective surgery, telephonic reviews for outpatients, telematic clinical committees and the designation of COVID19-specific areas; as well as to develop locally adapted protocols for COVID-19 screening and safe care of COVID-19 patients. Because of these measures, none of the healthcare workers present during the procedures was infected, and the positive cases among ward staff were anecdotal. Quick implementation of protective measures is essential to minimize infections in healthcare workers, which have risen to $20 \%$ in endemic countries such as Italy [10].

- In patients with SARS-CoV-2 infection, worse postoperative outcomes are related to the development of ARDS and respiratory failure, but fortunately, that did not occur in any of our patients $(0 \%)$, in contrast with the ARDS rates of other reported surgical series (75\% [2] and 32.4\% [3]). The rate of pneumonia was $57.1 \%$ in our series, being higher in patients who underwent less complex-grade $1 / 2$ - procedures $(75 \%)$ than in those who underwent grade $3 / 3+$ procedures $(50 \%)$, which probably contributed to the lower mortality rate. Infection by SARS-CoV-2 during the perioperative period was associated with a mortality rate of $7.14 \%$ in our series, even with a high percentage of complex procedures $(71.42 \%$ of grade $3 / 3+$ procedures). However, the small number of patients does not allow us to make generalized conclusions. Independently of unknown factors such as viral load and with a similar degree of complexity of the procedures, the $7.14 \%$ mortality rate in this series differs considerably from the mortality rates reported elsewhere for COVID-19 surgical patients, which are as high as $20.5 \%$ [3] (64.7\% for grade 3 and 4 procedures) and $75 \%$ [2]. Further studies are needed to determine which factors influence the morbidity and mortality of surgical patients who develop COVID-19 perioperatively.

Funding No funding was received for this work.

\section{Compliance with ethical standards}

Conflict of interest Ana Senent-Boza and the other co-authors declare no conflicts of interest.

\section{References}

1. Zhu N, Zhang D, Wang W, Li X, Yang B, Song J, et al. A novel coronavirus from patients with pneumonia in China, 2019. N Engl J Med. 2020;382(8):727-33.

2. Aminian A, Safari S, Razeghian-Jahromi A, Ghorbani M, Delaney CP. COVID-19 outbreak and surgical practice: unexpected fatality in perioperative period. Ann Surg. 2020. https://doi.org/10.1097/ SLA.0000000000003925(Epub ahead on print).

3. Lei S, Jiang F, Su W, Chen C, Chen J, Mei W, et al. Clinical characteristics and outcomes of patients undergoing surgeries during the incubation period of COVID-19 infection. E Clin Med. 2020. https ://doi.org/10.1016/j.eclinm.2020.100331(Epub ahead on print).

4. General surgery protocols for COVID-19. Virgen del Rocio university hospital, Seville, Spain. Available at: https://www.hospitaluv rocio.es/entrada-blog/protocolos-c-general-covid-19. Accesed April $12,2020$.

5. National guideline center (national institute for health and care excellence). Routine preoperative tests for elective surgery. 2016. Available at: https://www.ncbi.nlm.nih.gov/books/NBK355755/pdf/ Bookshelf_NBK355755.pdf. Accessed 12 Apr 2020.

6. Wu Z, McGoogan JM. Characteristics of and important lessons from the coronavirus disease 2019 (COVID-19) outbreak in China: summary of a report of 72314 cases from the Chinese center for disease control and prevention. JAMA. 2020;323(13):1239-42.

7. Ai T, Yang Z, Hou H, Zhan C, Chen C, Lv W, et al. Correlation of chest CT and RT-PCR testing in coronavirus disease 2019 (COVID19) in China: a report of 1014 cases. Radiology. 2020. https://doi. org/10.1148/radiol.2020200642(Epub ahead on print).

8. Zhou X, Li Y, Li T, Zhang W. Follow-up of asymptomatic patients with SARS-CoV-2 infection. Clin Microbiol Infect. 2020. https:// doi.org/10.1016/j.cmi.2020.03.024.

9. Instituto de Salud Carlos III. Guía para la utilización de test rápidos de anticuerpos para COVID-19 [Guidance on the use of rapid antibodies test for COVID-19]. 2020. Available at: https://www.mscbs .gob.es/profesionales/saludPublica/ccayes/alertasActual/nCov-China /documentos/Guia_test_diagnosticos_serologicos_20200407.pdf. Accessed 12 Apr 2020.

10. The LT. COVID-19: protecting health-care workers. Lancet (Lond Engl). 2020;395(10228):922.

Publisher's Note Springer Nature remains neutral with regard to jurisdictional claims in published maps and institutional affiliations. 